\title{
Gastrografin: A diagnostic and therapeutic agent
}

\author{
Authors: \\ Dr Adel Ekladious - MBCHB MD, FRCPI, FRCPG, FRACP, FACP \\ Mai Yamanaka - BMedSc \\ Dr. Luke Philip Wheeler, BAppSc (Hum Mvt), M Physio, MBBS (WA).
}

\section{Correspondence to Professor Adel Ekladious}

E-mail: ekladiou@hotmail.com

Tel.: +61499449905

\section{Declarations/ Competing Interests:}

None, including no funding received.

\section{Keywords:}

Gastrografin, obstruction, small bowel, therapeutic, adhesions

\begin{abstract}
$\underline{\text { Abstract }}$
Background: Published literature supports therapeutic Gastrografin use in adhesive small bowel obstruction.

Case presentation: An 81-year-old woman presented with 2 day history of persistent vomiting, nausea and abdominal pain. An abdominal computerised tomography (CT) revealed signs of small bowel obstruction, likely secondary to her history of endometriosis and ovario-hysterectomy. Gastrografin was administered and follow-up abdominal X-ray at 4 hours post administration, and CT abdomen at 20 hours post administration illustrated oral contrast through to the colon and relief of small bowel obstruction. Oral feeding was commenced 24 hours post administration and she was discharged home on a full diet.

Conclusion: Though Gastrografin for SBO is primarily used by surgical teams, its use by internal medicine teams as conservative management can obviate the need for surgical intervention. This case report highlights the value of Gastrografin in peripheral hospitals with limited access to emergency surgical services, and where surgical intervention is not possible or not advised due to medical comorbidities.
\end{abstract}




\section{Gastrografin: a diagnostic and therapeutic agent}

Gastrografin is a water-soluble, high osmolality contrast medium that has been used diagnostically to define, and therapeutically to relieve adhesive small bowel obstructions (SBO). Experimental and proof-of-concept studies have demonstrated that oral Gastrografin is both safe and effective at relieving up to $70 \%$ of adhesive SBO when administered in the first 24 hours. ${ }^{1}$ This is primarily due to its hyper-osmotic nature, as it promotes extra-luminal fluid shift into the bowel lumen, decreasing oedema and improving peristaltic bowel action. ${ }^{2}$ When used effectively, Gastrografin may negate the need for surgical intervention where surgery is not possible, or not readily available.

This case occurred in an Australian peripheral hospital with no emergency surgical services on-site (available via inter-hospital transfer). The patient was an independent high-functioning 81 year old female, with a past medical history of recent SBO (managed conservatively), endometriosis, breast and lung cancer, type 2 diabetes mellitus, hypertension and gastro-oesophageal reflux disease, who presented with a 2 day history of persistent vomiting, nausea and abdominal pain. An abdominal computerised tomography (CT) revealed dilatation of the small bowel proximal to the ileocaecal junction and a gradual transition point in the right iliac fossa (see figure 1). In the absence of abdominal metastases, this was suggestive of a recurrent SBO secondary to her history of endometriosis and ovario-hysterectomy, which is a frequent cause for this pathology in females. ${ }^{3}$

After management options were discussed with the patient, surgical intervention was declined due to her medical comorbidities. She was admitted as a medical patient to the acute services unit, and therapeutic Gastrografin was considered on discussion with the local tertiary hospital surgical team. As per protocol, a nasogastric tube was inserted and she was made nil-by-mouth initially during her admission. 
The patient was consented to the procedure and its risks (lethal pulmonary oedema if aspirated and anaphylactoid reactions) $)^{2}$. She was positioned upright to decrease risk of vomitus and aspiration, and then $100 \mathrm{ml}$ of Gastrografin was administered via nasogastric tube by slow push. After approximately 30 minutes the patient passed a large volume of Type 7 Bristol stool, progressing to Type 4 Bristol stools within 2 hours. Abdominal X-ray at 4 hours post administration, and CT abdomen at 20 hours post administration illustrated oral contrast through to the colon and relief of small bowel obstruction (Fig 1). Oral feeding was commenced 24 hours post administration and she was discharged home on a full diet.

Primarily used by surgical teams, we believe the use of Gastrografin for SBO by internal medicine teams is rare. Published literature demonstrates that if Gastrografin reaches the colon within 24 hours, patients were more frequently able to be managed conservatively, without the need for surgical intervention. ${ }^{4}$ This outcome is of particular importance in peripheral hospitals with limited access to emergency surgical services, and where surgical intervention is not possible or not advised due to medical comorbidities.

Despite the increasing volume of studies of therapeutic Gastrografin for SBO, further research is required to address potential therapeutic value for mechanical bowel obstruction and large bowel obstruction. ${ }^{5}$ 


\section{References}

${ }^{1}$ Cox MR, Gunn IF, Eastman MC, Hunt RF, Heinz AW. The safety and duration of non-operative treatment for adhesive small bowel obstruction. ANZ Journal of Surgery 1993; 63(5): 367-371.

${ }^{2}$ Choi HK, Chu KW, Law WL. Therapeutic value of Gastrografin in Adhesive Small Bowel Obstruction after unsuccessful conservative treatment. Annals of Surgery 2002; 236(1): 1-6.

${ }^{3}$ Ceglie AD, Bilardi C, Blanchi S, Picasso M, Muzio MD, Trimarchi A, et al. Acute small bowel obstruction caused by endometriosis: A case report and review of the literature. World Journal of Gastroenterology 2008; 14(21): 3430-3434.

${ }^{4}$ Chen SC, Lin FY, Lee PH, Yu SC, Wang SM, Chang KJ. Water-soluble contrast study predicts the need for early surgery in adhesive small bowel obstruction. British Journal of Surgery 1988; 85(12): 1692-1694.

${ }^{5}$ Syrmis W, Richard R, Jenkins-Marsh S, Chia SC, Good P. Oral water soluble contrast for malignant bowel obstruction (Protocol). Cochrane Database of Systematic Reviews 2015. 